Draft VERsion April 2, 2020

Typeset using LATEX twocolumn style in AASTeX61

\title{
THREE-DIMENSIONAL KINEMATIC RECONSTRUCTION OF THE OPTICALLY-EMITTING, HIGH-VELOCITY, OXYGEN-RICH EJECTA OF SUPERNOVA REMNANT N132D
}

\author{
Charles J. Law, ${ }^{1}$ Dan Milisavljevic, ${ }^{2}$ Daniel J. Patnaude,,${ }^{1}$ Paul P. Plucinsky,${ }^{1}$ Michael D. Gladders, ${ }^{3}$ \\ Judy Schmidt, ${ }^{4}$ Niharika Sravan, ${ }^{2}$ John Banovetz, ${ }^{2}$ Hidetoshi Sano, ${ }^{5,6}$ Jordan M. McGraw, ${ }^{7}$ \\ George Takahashi, ${ }^{7}$ and Salvatore Orlando ${ }^{8}$
}

\footnotetext{
${ }^{1}$ Center for Astrophysics | Harvard \& Smithsonian, 60 Garden St., Cambridge, MA 02138, USA

${ }^{2}$ Department of Physics and Astronomy, Purdue University, 525 Northwestern Avenue, West Lafayette, IN 47907, USA

${ }^{3}$ Department of Astronomy \&5 Astrophysics, The University of Chicago, 5640 South Ellis Avenue, Chicago, IL 60637, USA

${ }^{4}$ Astrophysics Source Code Library, Michigan Technological University, 1400 Townsend Drive, Houghton, MI 49931, USA

${ }^{5}$ Institute for Advanced Research, Nagoya University, Furo-cho, Chikusa-ku, Nagoya 464-8601, Japan

${ }^{6}$ Department of Physics, Nagoya University, Furo-cho, Chikusa-ku, Nagoya 464-8601, Japan

${ }^{7}$ Research Computing, ITaP, Purdue University, West Lafayette, IN 47907, USA

${ }^{8}$ INAF-Osservatorio Astronomico di Palermo "G.S. Vaiana", Piazza del Parlamento 1, I-90134 Palermo, Italy
}

(Received; Revised; Accepted)

Submitted to ApJ

\begin{abstract}
We present a three-dimensional kinematic reconstruction of the optically-emitting, oxygen-rich ejecta of supernova remnant N132D in the Large Magellanic Cloud. Data were obtained with the $6.5 \mathrm{~m}$ Magellan telescope in combination with the IMACS+GISMO instrument and survey [O III] $\lambda \lambda 4959,5007$ line emission in a $\sim 3^{\prime} \times 3^{\prime}$ region centered on N132D. The spatial and spectral resolution of our data enable detailed examination of the optical ejecta structure. The majority of N132D's optically bright oxygen ejecta are arranged in a torus-like geometry tilted approximately $28^{\circ}$ with respect to the plane of the sky. The torus has a radius of $4.4 \mathrm{pc}\left(D_{\mathrm{LMC}} / 50 \mathrm{kpc}\right)$, exhibits a blue-shifted radial velocity asymmetry of -3000 to $+2300 \mathrm{~km} \mathrm{~s}^{-1}$, and has a conspicuous break in its circumference. Assuming homologous expansion from the geometric center of O-rich filaments, the average expansion velocity of $1745 \mathrm{~km} \mathrm{~s}^{-1}$ translates to an age since explosion of $2450 \pm 195 \mathrm{yr}$. A faint, spatially-separated "runaway knot" (RK) with total space velocity of $3650 \mathrm{~km} \mathrm{~s}^{-1}$ is nearly perpendicular to the torus plane and coincident with X-ray emission that is substantially enhanced in Si relative to the LMC and N132D's bulk ejecta. These kinematic and chemical signatures suggest that the RK may have had its origin deep within the progenitor star. Overall, the main shell morphology and high-velocity, Si-enriched components of N132D have remarkable similarity with that of Cassiopeia A, which was the result of a Type IIb supernova explosion. Our results underscore the need for further observations and simulations that can robustly reconcile whether the observed morphology is dominated by explosion dynamics or shaped by interaction with the environment.
\end{abstract}

Keywords: Magellanic Clouds — ISM: kinematics and dynamics — ISM: individual objects (N132D) — ISM: supernova remnants - supernovae: general 


\section{INTRODUCTION}

Young, nearby supernova remnants (SNRs) offer rare opportunities to investigate kinematic and chemical details of supernova ( $\mathrm{SN}$ ) explosions at fine scales that are impossible to achieve in unresolved extragalactic events (Milisavljevic \& Fesen 2017). Their morphology and elemental distribution provide unique insight into where and how asymmetry is introduced during core collapse (Lopez \& Fesen 2018), which is presently a key area of investigation in increasingly sophisticated 2D and 3D simulations (Müller \& Janka 2015; Janka et al. 2016; Kuroda et al. 2017; O'Connor \& Couch 2018; Burrows et al. 2019). Expanding stellar ejecta that encode valuable information about the explosion dynamics, nucleosynthetic yields, and mixing of the progenitor star can be resolved, measured, and tracked. The progenitor star system's transitions through evolutionary stages leading up to core collapse and associated mass loss can also be explored by studying the SNR's interaction with its surrounding environment.

O-rich SNRs are particularly well-suited for studies of core-collapse SN explosion dynamics and offer powerful tests of simulations that are now able to evolve from SN to the remnant phase (Orlando et al. 2015, 2016; Ferrand et al. 2019). This, in part, is because they are often associated with progenitor stars that were largely stripped of their hydrogen envelopes. SNe of this variety (Type IIb, Ib, Ic; see Gal-Yam 2017 for details) retain critical elements of their central explosion dynamics that are otherwise disrupted in H-rich explosions (Milisavljevic et al. 2010). Only a handful of O-rich remnants have so far been identified in our Galaxy and in nearby galaxies. Cassiopeia A (Cas A), which is regarded as the prototypical O-rich SNR (Kirshner \& Chevalier 1977; Chevalier \& Kirshner 1978), Puppis A (Winkler \& Kirshner 1985), and G292+1.8 (Goss et al. 1979; Murdin \& Clark 1979) are among the Galactic members. N132D (Danziger \& Dennefeld 1976) and 0540-69.3 (Mathewson et al. 1980) are in the Large Magellanic Cloud, and 1E 0102.2-7219 (E0102; Dopita et al. 1981), 0103-72.6 (Park et al. 2003), and B0049-73.6 (Hendrick et al. 2005) are in the Small Magellanic Cloud. Several mixedmorphology SNRs, including CTB 1, HB 3, and W28 have also shown evidence of O-rich ejecta (Lazendic \& Slane 2006; Pannuti et al. 2010, 2017).

Located in the stellar bar of the LMC, N132D is the brightest X-ray and gamma-ray emitting SNR in that galaxy (Clark et al. 1982; Hwang et al. 1993; Favata et al. 1997; Borkowski et al. 2007; H.E.S.S. Collaboration et al. 2015; Ackermann et al. 2016). The remnant nature of N132D was first recognized by Westerlund \& Mathewson (1966), who noticed an association between a non-thermal radio source and bright [S II] optical emission. The discovery of high-velocity [O III] and [Ne III] ejecta established the relative youth of N132D, indicating that the reverse shock has not yet reached the center of the remnant (Danziger \& Dennefeld 1976; Lasker 1978). Subsequent optical/UV spectra from the Hubble Space Telescope (HST) show strong emission from C- and Ne-burning elements but little emission from Oburning elements (e.g., Morse et al. 1996; France et al. 2009), which indicates that the remnant may be the debris of a $\sim 25 \mathrm{M}_{\odot}$ progenitor star that experienced significant mass loss and exploded as a Type Ib SN (Blair et al. 2000).

High-resolution X-ray images reveal a horseshoeshaped forward shock (e.g., Borkowski et al. 2007; Xiao \& Chen 2008; Bamba et al. 2018), which has been modeled as a blast wave impacting an irregular cavity wall (Chen et al. 2003), while spatially resolved X-ray spectroscopy reveals an asymmetric Fe-K distribution from an estimated $15 \pm 5 \mathrm{M}_{\odot}$ progenitor (Sharda et al. 2020, submitted). This outer shell is also detected in hot dust emission in $24 \mu \mathrm{m}$ Spitzer maps (Tappe et al. 2006), and submillimeter observations suggest a physical and kinematic association between the southern portion of N132D's outer shell and nearby, likely natal, CO molecular clouds (Banas et al. 1997; Sano et al. 2017; Sano in prep.). Numerous luminous, shocked dense clouds with normal LMC ISM abundances (e.g., Blair et al. 2000; Dopita et al. 2018) and velocity dispersions of $\sim 200 \mathrm{~km} \mathrm{~s}^{-1}$ are present throughout N132D. Prominent among these clouds are the outer western edge of the remnant and Lasker's bowl (Lasker 1980), which is located in the northern part of the remnant and exhibits the curved inner boundary of a classical bow shock morphology. Since these clouds do not show enhancements in $[\mathrm{N} \mathrm{II}]$, they were likely pre-existing ISM clouds over which the SNR blast wave recently passed (Dopita et al. 2018).

The optical remnant is approximately $110^{\prime \prime}$ in diameter and has three distinct emission components: (1) high-velocity filaments rich in oxygen and neon but lacking hydrogen, (2) low-to-moderate-velocity H-rich knots and filaments, consistent with clumpy mass loss by the progenitor star, and (3) diffuse emission from normal LMC abundance clouds at rest with respect to the local medium. Several Doppler reconstructions of N132D's O-rich optical material have been conducted over the last four decades. The first attempts revealed a thin, inclined ring (Lasker 1980), while later studies claimed that the O-rich knots instead form a thin shell (Morse et al. 1995). A total velocity range of $\sim 4500 \mathrm{~km} \mathrm{~s}^{-1}$ was observed in the O-rich knots with blue-shifted ma- 


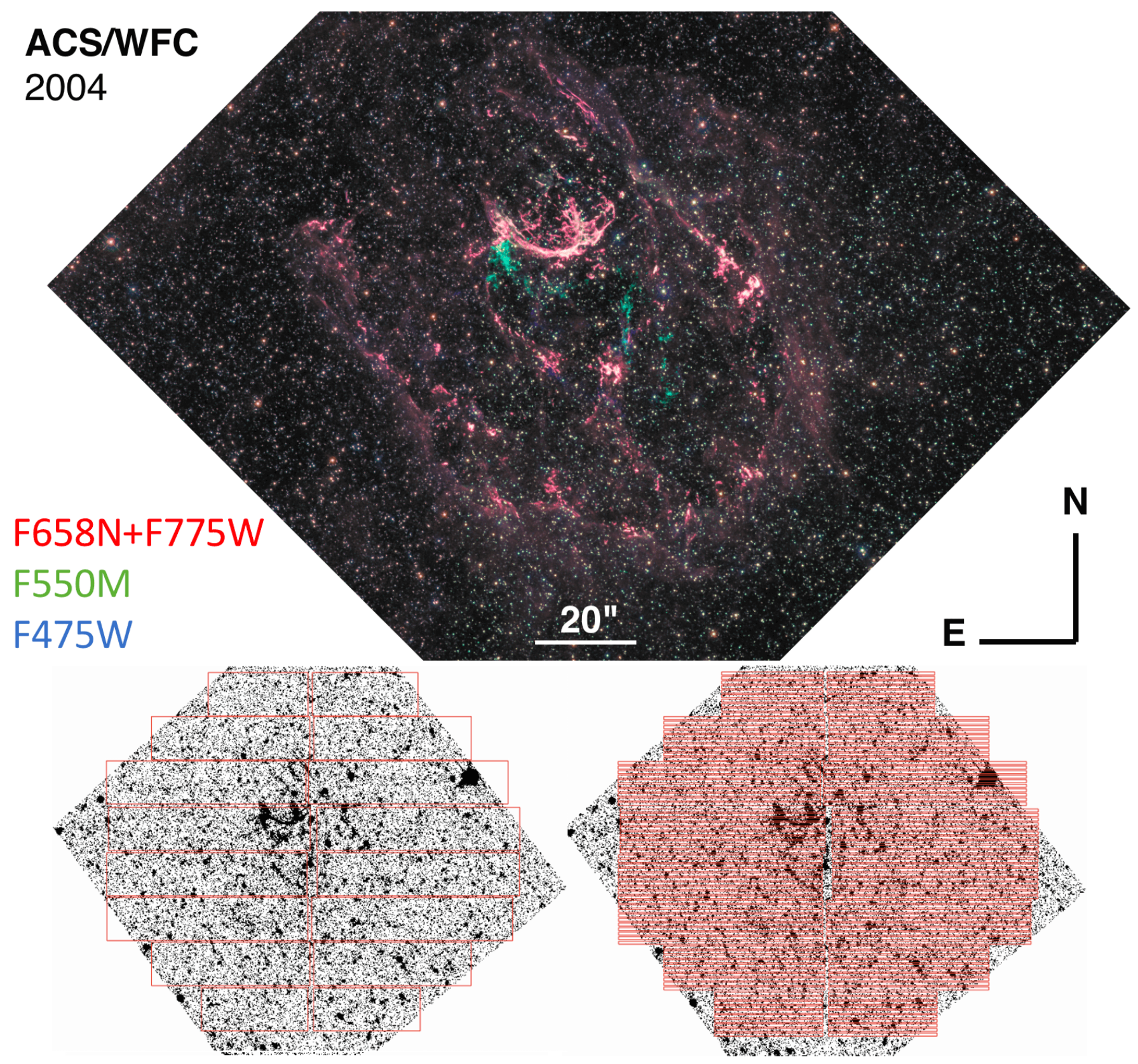

Figure 1. Top: HST ACS composite image of N132D sensitive to oxygen and hydrogen emission using F475W, F550M, and F658N+F775W filters in blue, green, and red, respectively. Filamentary blue and green emission represents the location of O-rich ejecta, while diffuse red emission traces the horseshoe-shaped forward shock and ISM/CSM-related structures.Bottom left: GISMO footprint on N132D. Bottom right: Finding chart of all long-slit positions. Background image is the HST/ACS F475W image. Data were retrieved from the Mikulski Archive for Space Telescopes and are associated with Proposal 12001 (PI J. Green).

terial having higher velocities than the redshifted ejecta (e.g., Morse et al. 1995; Vogt \& Dopita 2011). A survey of [O III] $\lambda 5007$ dynamics by Vogt \& Dopita (2011) revealed that the majority of O-rich ejecta form a ring $\sim 12 \mathrm{pc}$ in diameter and are inclined at an angle of $\sim 25^{\circ}$ to the line of sight. Vogt \& Dopita (2011) suggest that this ring of O-rich material is from ejecta in the equatorial plane of a bipolar explosion and that the observed morphology of N132D was heavily influenced by the pre-SN mass loss from the progenitor star. Furthermore, Vogt \& Dopita (2011) interpret the "runaway knot" (RK), which was first recognized by Morse et al. (1995) as an outlying O-rich ejecta clump with higherthan-average space velocity, as possible evidence of a polar jet. 


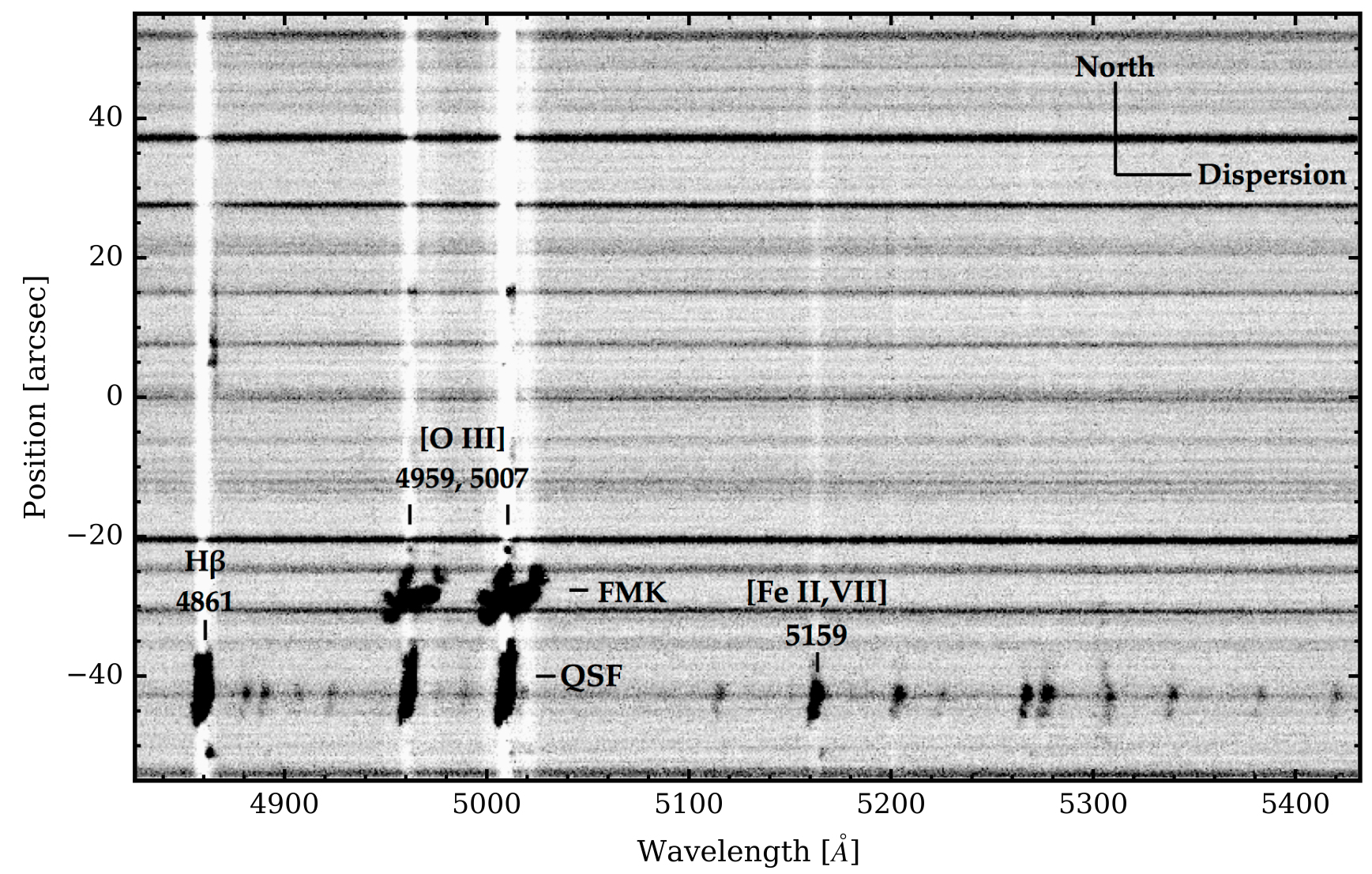

Figure 2. Example of a fully-reduced and cleaned 2D spectrum of a slit position along the main shell from which $1 \mathrm{D}$ extractions were made. Horizontal lines represent continuum emission from stars contained within the slit. Fast moving knots (FMKs), the focus on this study, and ISM/CSM-related quasi-stationary flocculi (QSF) are labeled. Wavelength positions $(\AA)$ for the brightest emission lines are marked.

Unlike other young SNRs such as E0102 (Finkelstein et al. 2006) and Cas A (Fesen 2001; Fesen et al. 2006), N132D lacks proper motion measurements of its ejecta. Hence, age estimates have been based on radial velocities that are translated into expansion velocities and the overall angular extent of the filamentary structures. From the dynamics of fast-moving $\mathrm{O}$ and Ne material, Danziger \& Dennefeld (1976) estimated a maximum age of $3440 \mathrm{yr}$ and a probable age of $\sim 1350 \mathrm{yr}$. Lasker (1980) derived a similar age of $\sim 1300 \mathrm{yr}$, while subsequent determinations from Morse et al. (1995) and Sutherland \& Dopita (1995) found ages of $\sim 3150 \mathrm{yr}$ and $\sim 2350 \mathrm{yr}$, respectively. More recently, Vogt \& Dopita (2011) estimated an age of $2500 \mathrm{yr}$. The inconsistency in these age estimates is directly related to differing assumptions about the shape of the O-rich ejecta.

To resolve this discrepancy in remnant age estimates that range between 1300-3440 yr and to explore N132D's morphology in hopes of gaining insight into the dynamics of the original supernova explosion, we undertook a detailed spectroscopic mapping of the en- tire N132D remnant with higher spatial resolution than previous efforts. We describe the observations and our data reduction methods in Sections 2 and 3, respectively. In Section 4, we discuss how these data were used to develop a 3D kinematic reconstruction of the optically-emitting oxygen ejecta and present the results of our reconstruction. In Section 5, we discuss the kinematic properties of these ejecta in the context of SN explosion processes and the ejecta structure of other well-studied SNRs. We then summarize our findings in Section 6.

\section{OBSERVATIONS}

In Figure 1, we present a composite image of N132D from high spatial resolution $H S T$ imaging taken with the Advanced Camera for Surveys (ACS) that maps the optical emission of N132D. These images, which were obtained on 2004 January 22, provide complete spatial coverage of the remnant (Beasley et al. 2004) and are associated with Proposal 12001 (PI J. Green). The ACS Wide Field Channel (WFC) F475W filter (SDSS $g$ ) cov- 

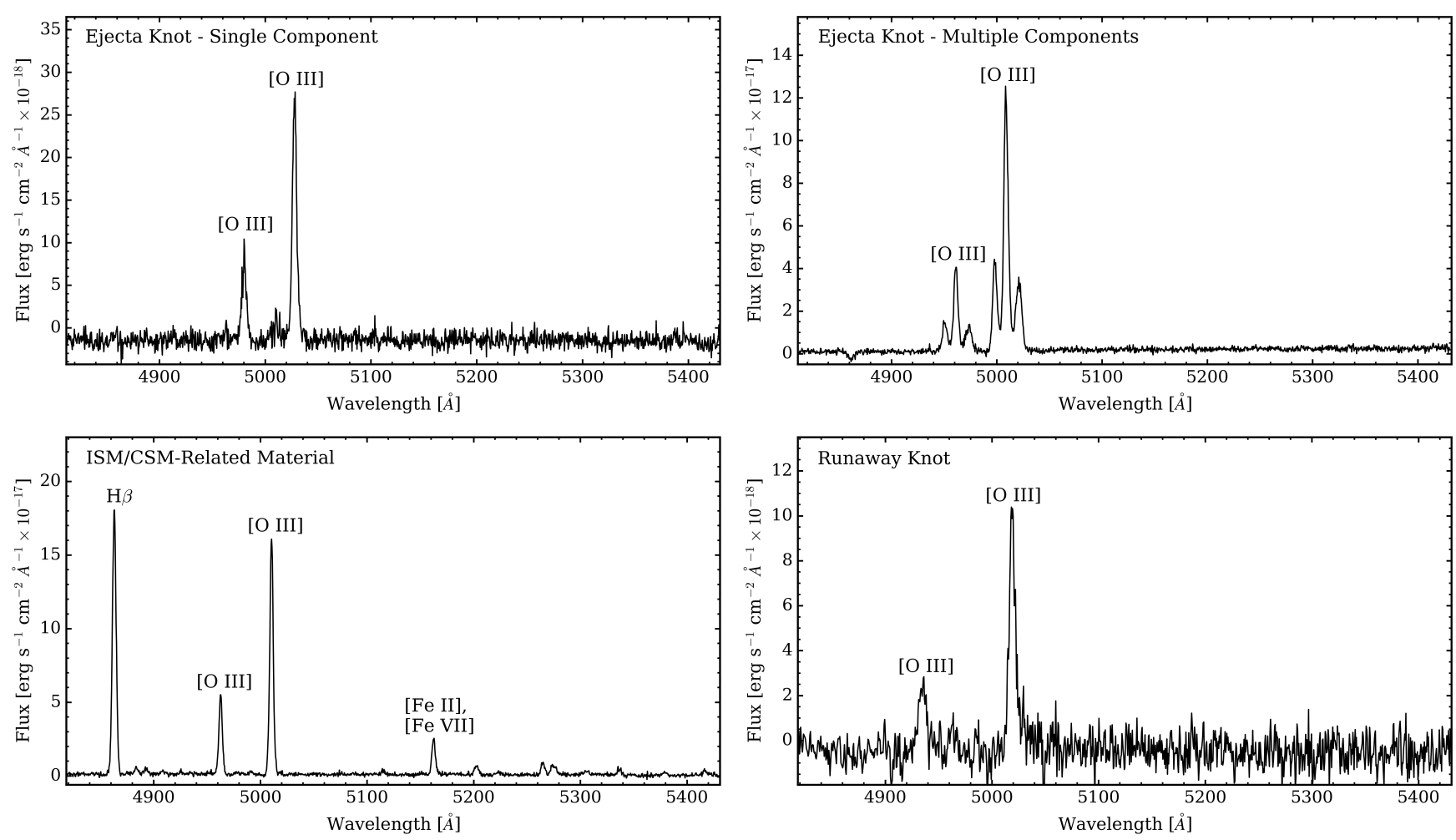

Figure 3. Examples of 1D spectra of ejecta knots of N132D used in the Doppler reconstruction. The top left spectrum from an ejecta knot shows a single velocity component, while the top right shows a more complicated ejecta knot with three separate velocity components. Bottom left shows ISM/CSM-related material, as indicated by numerous Fe lines and prominent $\mathrm{H} \beta$ emission. Bottom right presents a spectrum from the runaway knot, identifying it as bona fide O-rich ejecta.

ers the full range of Doppler shifted [O III] $\lambda \lambda 4959,5007$ emission from high-velocity, O-rich ejecta. The F550M filter is sensitive to continuum emission. The F658N filter is sensitive to shocked ambient medium emitting strongly in $\mathrm{H} \alpha$. The F775W filter ( $\operatorname{SDSS} i$ ) is sensitive to ambient medium emission lines of [Fe II], [Ca II], and [O II] (see, e.g., Dopita et al. 2018), with a small fraction of emission due to O-rich ejecta weakly emitting in [O II] $\lambda \lambda 7319,7330$.

Low-dispersion long-slit optical spectra across the entire supernova remnant N132D were obtained on 31 December 2015 and 1 January 2016 with the Inamori Magellan Areal Camera and Spectrograph (IMACS; Dressler et al. 2011) on the $6.5 \mathrm{~m}$ Baade Magellan telescope at Las Campanas Observatory. The $\mathrm{f} / 4$ camera was used with the Gladders Image-Slicing Multislit Option $(\mathrm{GISMO})^{1}$ in combination with a 600 lines $\mathrm{mm}^{-1}$ grating, the GISMO-3 filter $\left(\lambda_{\circ}=4981 \AA ; \Delta \lambda=947 \AA\right)$ and a mask of long-slits oriented east-west. Slit widths were fixed at $1.6^{\prime \prime}$ and slit lengths ranged from $57^{\prime \prime}$ to

\footnotetext{
1 http://www.lco.cl/telescopes-information/magellan/ instruments/imacs/gismo/gismoquickmanual.pdf
}

$110^{\prime \prime}$, depending on slit location within the GISMO footprint. The resulting dispersion was $0.378 \AA$ per pixel with an effective wavelength window of approximately 4810-5430 $\AA$ and a spectral resolution full-width-halfmaximum (FWHM) of $5.5 \AA$. The telescope was moved in the north-south direction in non-overlapping increments of $2.4^{\prime \prime}$ in order to cover the majority of the remnant $(67 \%)$. A finding chart of the GISMO footprint along with all long-slit positions is shown in Figure 1. At each position, exposures of length $2 \times 800 \mathrm{~s}$ were taken. Wavelength calibration He-Ne-Ar lamps were taken every two positions to track instrument flexure. The airmass was between 1.3-1.6 for all observations and the seeing was variable from $0.7^{\prime \prime}$ to $1.0^{\prime \prime}$. The spectrophotometric standard star LTT 3864 was observed as a flux calibration source.

\section{DATA REDUCTION}

Data reduction closely followed the methods of Milisavljevic \& Fesen (2013). A series of scripts were written to automate IRAF/PyRAF ${ }^{2}$ procedures to homoge-

\footnotetext{
${ }^{2}$ IRAF is distributed by the National Optical Astronomy Observatories, which are operated by the Association of Universi-
} 
neously reduce the spectral data. For each position, 2D images were trimmed, bias-subtracted, flattened, and co-added to remove cosmic rays. The L.A.Cosmic task (van Dokkum 2001) was employed to remove any residual cosmic rays. Images were then wavelength calibrated in the dispersion axis using comparison lamp images and straightened in the spatial axis using tracings of stellar continua.

Robust background subtraction was achieved with the IRAF task background using a sixth-order Chebyshev function fit along the spatial direction that was sampled from each individual pixel (i.e., no median pixelbinning). This resulted in sky emission-cleaned 2D images from which 1D spectra could then be extracted. An example of a fully-reduced and cleaned 2D image is shown in Figure 2.

Each line of the 2D spectra was extracted in regions of conspicuous [O III] $\lambda \lambda 4959,5007$ that were lacking in prominent $\mathrm{H} \beta$ emission. This selection targeted O-rich ejecta. No attempt was made to catalog $\mathrm{H} \beta$ emission associated with H-rich ISM/CSM. From these 1D extractions, knot velocities were measured through crosscorrelation of templates using the IRAF task xcsao. The template was constructed by assuming a 3:1 intensity ratio of the [O III] doublet (Storey \& Zeippen 2000). We present example spectra of several types of ejecta knots encountered in Figure 3. The xcsao task was run iteratively on all extracted 1D spectra for Doppler velocities ranging between -3000 and $+3000 \mathrm{~km} \mathrm{~s}^{-1}$ to identify individual knots. The spectra were first smoothed with a 7 by 7 boxcar before running the autocorrelation. This procedure was found to work well even in cases with multiple knots (e.g., Figure 3, top right), and a detailed example of this analysis is shown in Figure 4. Measured velocities are believed to be accurate to $\pm 30 \mathrm{~km} \mathrm{~s}^{-1}$ at the $68 \%$ confidence level.

Results of the preliminary selection were manually inspected for false positives. It was important to only select emission that could be sensibly associated in 3D kinematic space. The Lasker's bowl region of the remnant and other modest-velocity ejecta knots $( \pm 400 \mathrm{~km}$ $\mathrm{s}^{-1}$ ) required additional care owing to the significant presence of $\mathrm{H} \beta$, and these data are considered separately than the more secure O-rich high-velocity knot detections. Regions where emission from O-rich knots were identified are shown in Figure 5 and are labeled according to the notation from Morse et al. (1995). We

ties for Research in Astronomy, Inc., under cooperative agreement with the National Science Foundation. PyRAF is a product of the Space Telescope Science Institute, which is operated by AURA for NASA. refer the interested reader to Blair et al. (2000) and Dopita et al. (2018) for more detailed discussions of the spectroscopic properties of these types of knots.

A 8.3-magnitude earthquake struck approximately 500 miles south of Las Campanas Observatory on 16 September 2015 that disrupted optical alignment of GISMO within IMACS. There was no loss in data quality or coverage of the remnant, but the disruption did compromise use of pre-written pipeline procedures that stitch together the reformatted image slices. A variety of cross-checks ensured that consistent and accurate coordinates in right ascension (R.A.) and declination (Dec.) were assigned to radial velocity measurements for individual knots. Stars with well-measured coordinates encountered during the progression of long-slit positions provided fiducial reference points. Final fine-grain corrections to all positions were accomplished through comparison with high-resolution HST images (Figure 1) and overlays of bright stars from the 2MASS database (Skrutskie et al. 2006), which provided accurate positional offsets. Positional uncertainties are estimated to be no more than $1.6^{\prime \prime}$, which is on the order of the slit width.

\section{RESULTS}

\subsection{D Doppler Reconstruction}

A three-dimensional reconstruction of ejecta from a debris field requires knowledge of the center of expansion (COE). No proper motion analysis of N132D providing a $\mathrm{COE}$ from direct measurements of ejecta has been published. N132D has two suggested COEs inferred by different geometric assumptions: $\alpha(2000.0)=$ $5^{h} 25^{m} 02 .^{s} 7 ; \delta(2000.0)=-69^{\circ} 38^{\prime} 34^{\prime \prime}$, which marks the center of the O-rich knots assuming a symmetric distribution, and $\alpha(2000.0)=5^{h} 25^{m} 01 .{ }^{s} 4 ; \delta(2000.0)=$ $-69^{\circ} 38^{\prime} 31^{\prime \prime}$, which corresponds to the remnant center determined from fitting an ellipse to the diffuse outer rim (Morse et al. 1995). Figure 5 shows the location of these centers with respect to our observations. As this study is focused on O-rich knots, we assume N132D's explosion center to be located at the center of a symmetric distribution of O-rich ejecta. The two reported centers are separated by $\sim 7^{\prime \prime}$, thus the choice between these COEs does not impact broader conclusions about the kinematics and morphology of N132D.

To simplify reconstruction, we also assume that the fast-moving O-rich ejecta follow ballistic trajectories from the COE of the initial SN explosion. Even without proper motions, this is a reasonable assumption to make since individual O-rich knots are originally very dense compared to the surrounding ISM/CSM $(\chi>100)$ and the cloud shock velocities $\left(\sim 100 \mathrm{~km} \mathrm{~s}^{-1}\right)$ are much 

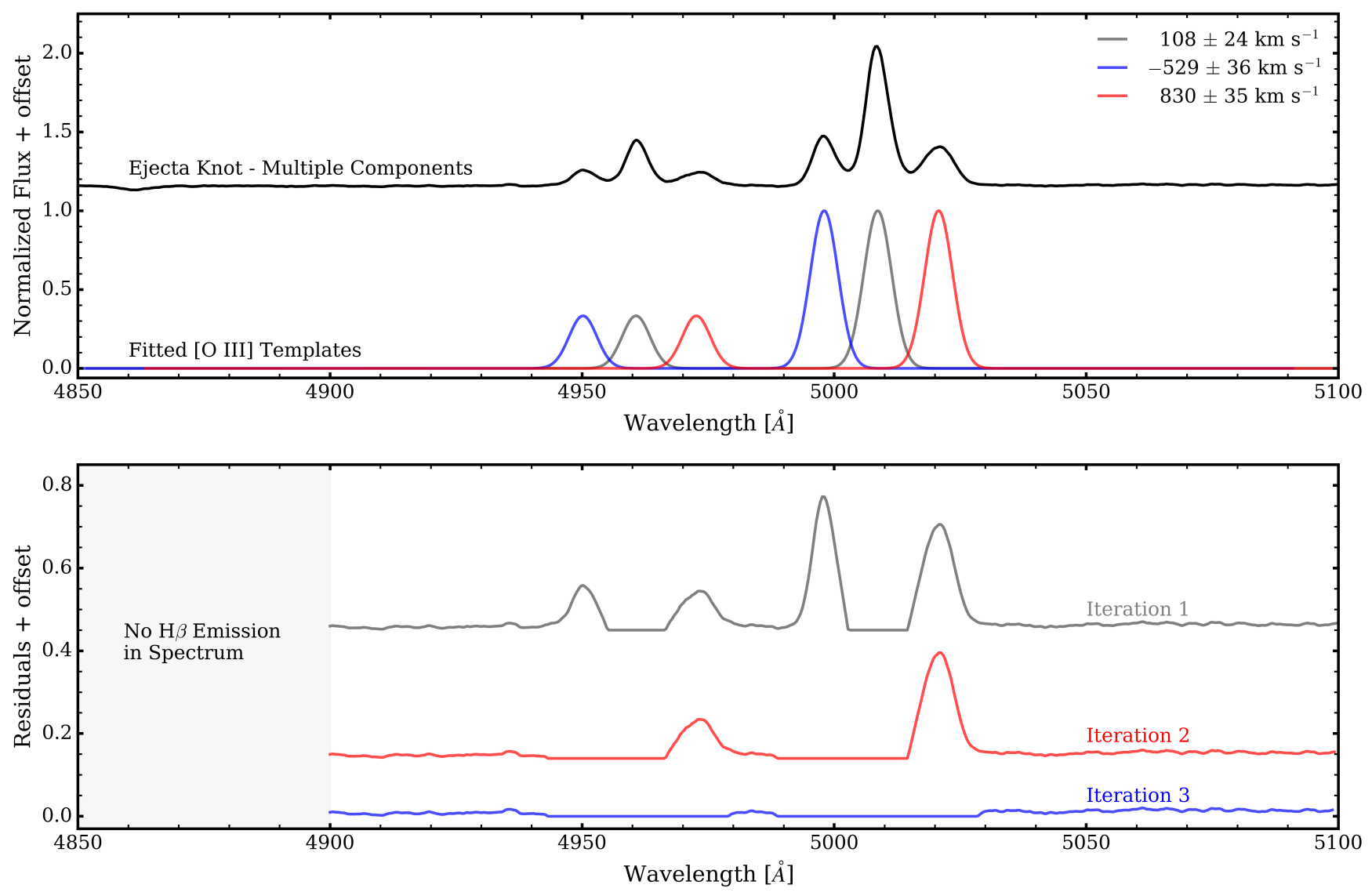

Figure 4. Top: An example of the cross-correlation method used to derive radial velocities of an O-rich ejecta knot with multiple velocity components. The ejecta spectrum has been smoothed, as described in the text, and is shown in black, while the three fitted [O III] doublet templates are shown below in blue, gray, and red. Bottom: Residuals for three iterations of the autocorrelation fitting process.

smaller than the typical O-rich ejecta velocities $(\sim 1000-$ $2000 \mathrm{~km} \mathrm{~s}^{-1}$ ). Such ballistic trajectories from the COE are described by the simple relation:

$$
v=r / S
$$

where $v$ is the radial velocity, $r$ is the angular distance from the explosion center, and $S$ is the scaling factor ${ }^{3}$. We adopt a distance of $d=50 \mathrm{kpc}$ to the LMC (Panagia et al. 1991; van der Marel et al. 2002), which implies a linear scale of $0.24 \mathrm{pc}$ arcsecond $^{-1}$.

We determine the value of $S$ by fitting the measured Doppler velocities to a spherical expansion model. We closely follow the procedures reviewed in Milisavljevic \& Fesen (2013) and also discussed in Reed et al. (1995) and DeLaney et al. (2010). In brief, we fit a semi-circle model

\footnotetext{
${ }^{3}$ We note that Equation 1 is misprinted as multiplication of $S$ and $r$ in Milisavljevic \& Fesen (2013), instead of division, as is correctly reported here.
}

to the velocity distribution and the observed projected radius. We parameterize the model as:

$$
\left(r_{p} / S\right)^{2}+\left(v_{D}-v_{c}\right)^{2}=\left(v_{c}-v_{m}\right)^{2},
$$

where $v_{c}$ is the center of the velocity distribution, $v_{m}$ is the minimum velocity at which the semi-circle crosses the velocity axis, $r_{p}$ is the observed projected radius, $v_{D}$ is the observed Doppler velocity, and $S$ is a scaling factor relating the velocity axis to the spatial axis.

The results of our least-squares fit to the data are shown in Figure 6. The calculated Doppler velocities are $v_{c}=-203 \pm 20 \mathrm{~km} \mathrm{~s}^{-1}$ and $v_{m}=-1947 \pm 150 \mathrm{~km} \mathrm{~s}^{-1}$, and the scaling factor is $S=0^{\prime \prime} .010 \pm 0^{\prime \prime} .0005$ per $\mathrm{km} \mathrm{s}^{-1}$. We fit the expansion model using only the secure detections of main shell O-rich knots, excluding the RK and lower-velocity material potentially associated with ISM/CSM-related clouds. Similarly, we restrict all further analysis to these secure detections; while we did not include the RK in the fitting process, we do include it in the subsequent velocity-space reconstructions. Knot 

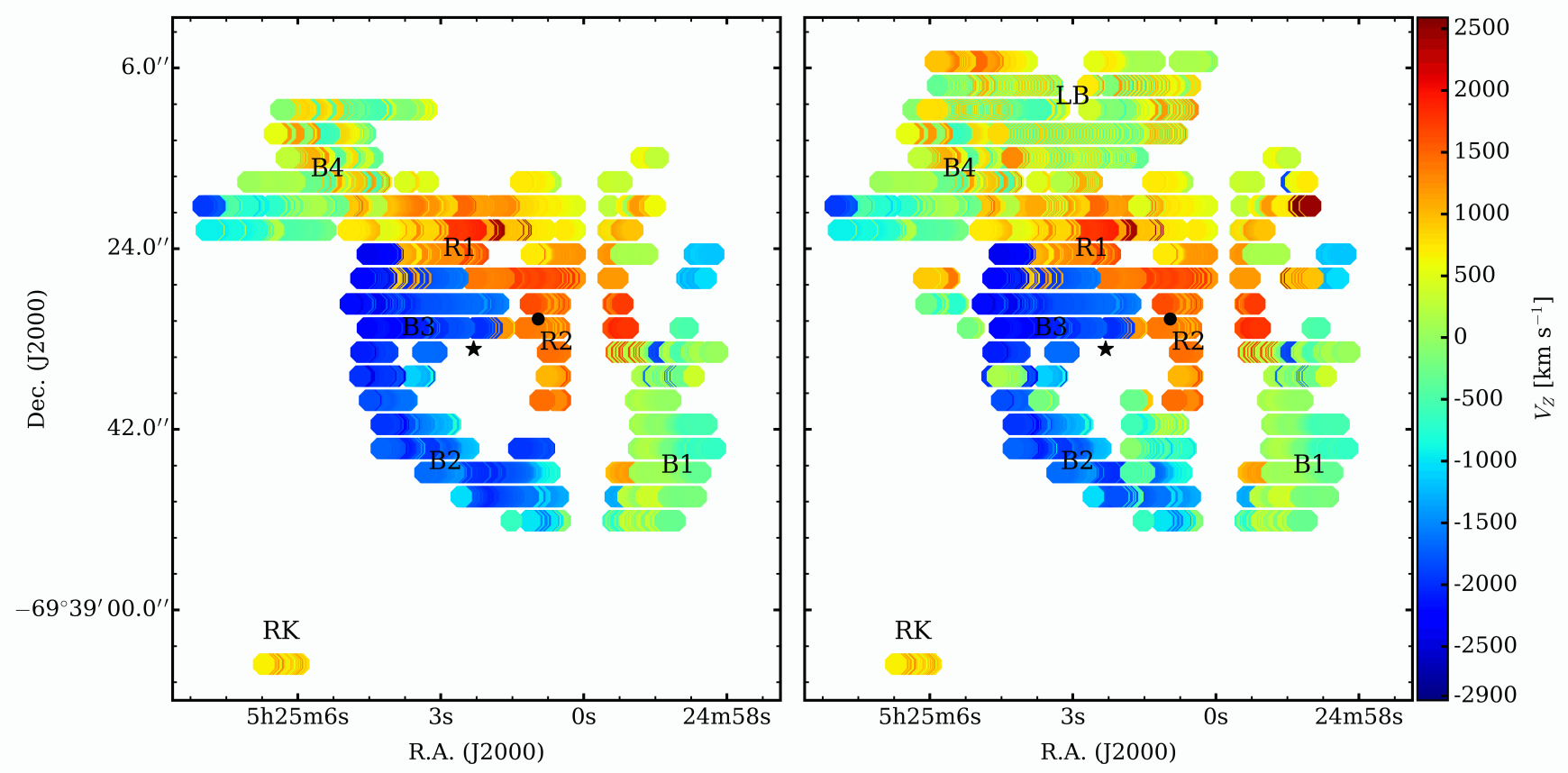

Figure 5. Regions in N132D where O-rich knot emission was detected. Major knots from Morse et al. (1995) have been identified along with the runaway knot $(\mathrm{RK})$ discussed in the text. Both proposed centers of the remnant from Morse et al. (1995) have been plotted, namely the center of the O-rich knots as a star $(\star)$ and that determined by fitting the remnant's outer rim as a filled black dot $(\cdot)$. Left: Only secure detections of pure O-rich knots are shown. Right: Both secure detections as well as slow-velocity knots and those spatially associated with ISM/CSM-related clouds such as Lasker's Bowl (LB) (Morse et al. 1996) are shown. Pixels are not to scale and have been enlarged for visual clarity.

positions in R.A. and Dec. were scaled to velocities using Equation 1. We used the COE and the calculated Doppler velocity $v_{c}$ to define a $3 \mathrm{D} \mathrm{COE}$ in velocity space from which all reported vector trajectories originate.

\subsection{Age Estimation}

From our fitting in the previous subsection, the projected radius of $\mathrm{N} 132 \mathrm{D}$ is $18.2^{\prime \prime}$, or $4.4 \mathrm{pc}$ at the LMC distance of $50 \mathrm{kpc}$. In velocity space, this radius corresponds to a projected velocity of $1745 \mathrm{~km} \mathrm{~s}^{-1}$. Knowing the distance and the rate of expansion (assuming ballistic trajectories), we are able to estimate the age of the remnant using

$$
r_{\mathrm{vel}}\left[\mathrm{km} \mathrm{s}^{-1}\right] \times \mathrm{t}=\mathrm{r}_{\text {dist }}[\mathrm{pc}],
$$

where $r_{\text {vel }}$ is the projected radius in $\mathrm{km} \mathrm{s}^{-1}, t$ is time of expansion, and $r_{\text {dist }}$ is the projected radius in pc. The time in terms of years is then given by:

$$
t[\mathrm{yr}]=9.785 \times 10^{5} \times\left(\mathrm{r}_{\mathrm{dist}}[\mathrm{pc}] / \mathrm{r}_{\mathrm{vel}}\left[\mathrm{km} \mathrm{s}^{-1}\right]\right)
$$

From these fitting results, we find an age of $2450 \pm 195$ yr, which is consistent with the previously-determined kinematic age of $2500 \mathrm{yr}$ from Vogt \& Dopita (2011). Our age estimate is also consistent with recent $\mathrm{X}$-ray
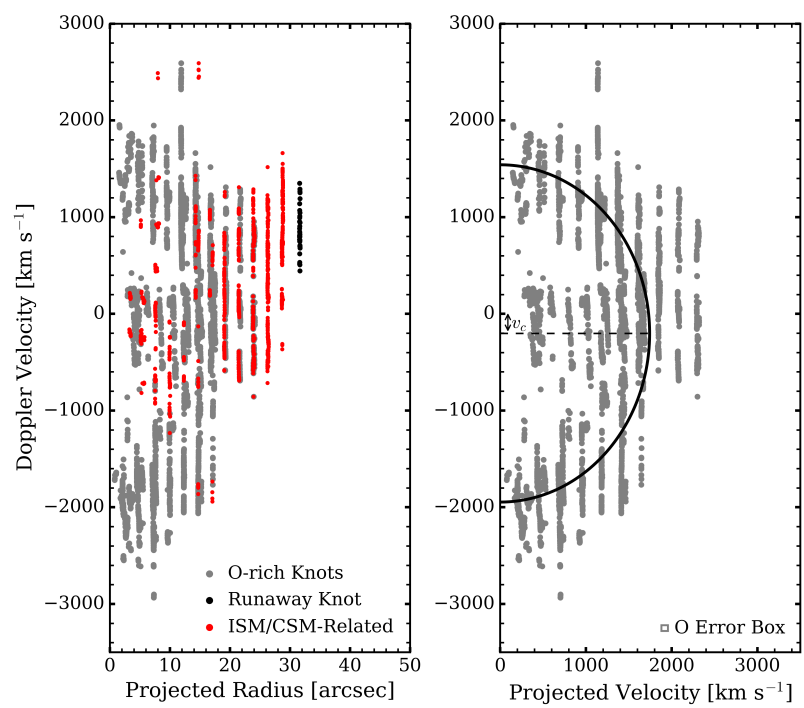

Figure 6. Left: Measured [O III] ejecta velocities versus projected radii from the COE. O-rich ejecta knots are shown in gray, the runaway knot in black, and ISM/CSM-related material in red. Right: Measured ejecta velocities versus projected radii, which have been converted to velocities using scaling factor $S=0^{\prime \prime} .010 \pm 0^{\prime \prime} .0005$ per $\mathrm{km} \mathrm{s}^{-1}$. Best-fit semi-circle is shown as a solid black line.

and gamma ray observations indicating that N132D is 


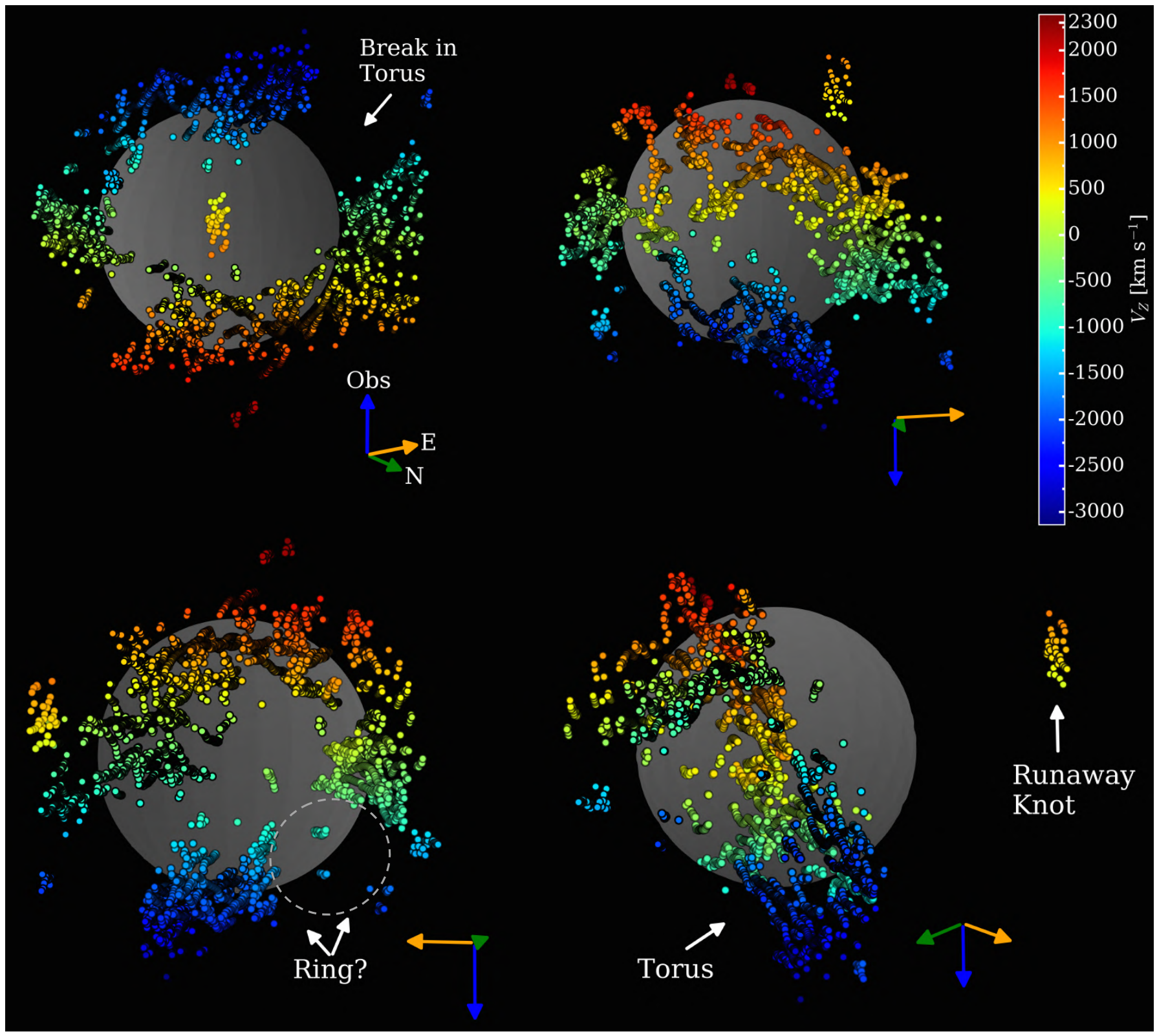

Figure 7. 3D Doppler reconstruction of N132D's optically-emitting, O-rich ejecta. The top left panel shows a clearer view of the broken torus structure. The top right and bottom left panels show two $45^{\circ}$ views on each side of N132D. The bottom right panel presents a side profile of the torus, which highlights the orthogonal position of the runaway knot with respect to the plane of the tilted ring. A translucent sphere is a visual aid to help distinguish between front and back material.

undergoing the transition from a young to middle-aged SNR (Bamba et al. 2018).

Compared to Vogt \& Dopita (2011), our ring diameter is $\sim 30 \%$ percent smaller than their value of $\approx 12 \mathrm{pc}$, but our age estimates are consistent. The discrepancy in diameter is not unexpected, however, given that Vogt \& Dopita (2011) manually adjusted the expansion time in order to visually reproduce a ring morphology. Vogt \& Dopita (2011) reasoned that if the remnant is a true ring, its projected size in the $\mathrm{X}-\mathrm{Z}$ plane should equal its major axis in the $\mathrm{X}-\mathrm{Y}$ plane and was the criterion from which they derived their age estimate. Our method calculates the ring diameter using an average of all points measured from data having higher spatial resolution and improves the overall fitting procedure. If we instead only fit the outer limb of the tilted ring in our data set, we also find a diameter of $\approx 48^{\prime \prime}(12 \mathrm{pc}$ ). Alternatively, if we fit only the inner limb of this ring, we would find a diameter of $\approx 24^{\prime \prime}(6 \mathrm{pc})$.

We found a conspicuous lack of O-rich ejecta with radial velocities between -1000 to $-2000 \mathrm{~km} \mathrm{~s}^{-1}$ and projected velocities of $\sim 2000 \mathrm{~km} \mathrm{~s}^{-1}$ (Figure 6). We 
investigated whether there were any significant gaps in our coverage at or near this phase space that would adversely affect our least squares fit of the ring diameter. We confirmed that there were no such gaps in our coverage. Instead, the absence of such data points is either the result of faint [O III] knots that were not detected at the sensitivity of our survey, or is related to intrinsic inhomogeneities in the spatial distribution of O-rich ejecta. We favor this latter explanation, especially since ejecta with radial velocities in this range are found to be associated with an incomplete ring-like structure toward the blue-shifted side of N132D (see Section 4.3.1.)

\subsection{O-rich Ejecta}

The results from our survey of N132D encompass 4126 individual data points with 4093 corresponding to main shell ejecta and 33 for the RK. Measured knot radial velocities are shown in Figure 7 with a color-coded gradient. The resulting Doppler maps represent the most complete catalog of its [O III] optically-emitting ejecta to date.

An animation of the radial velocity point cloud that has been enhanced with a surface model is provided in the supporting material for this paper. The surface model is first created using the Marching Cubes method and further retopologized with Catmull-Clark smoothing. The Marching Cubes algorithm uses an input threshold to polygonize a three-dimensional scalar field using a table of predefined facet configurations (Lorensen \& Cline 1987). Catmull-Clark operates by subdividing the surface of a mesh into smaller polygons and readjusting the vertices upon weighted averages (Catmull \& Clark 1998). By performing such a surface reconstruction, we aim to produce a more faithful reconstruction of the gas distribution, and thus a more physically accurate $3 \mathrm{D}$ representation of $\mathrm{N} 132 \mathrm{D}$. A demonstration of this surface reconstruction process is shown in Figure 8 .

Below, we discuss some specific characteristics of N132D that are revealed by our high-resolution 3D reconstruction of the remnant's kinematic structure.

\subsubsection{Kinematic Structure}

The majority of O-rich knots define a broken and distorted torus. The large-scale morphology is consistent with the 3D models presented in Vogt \& Dopita (2011) based on [O III] $\lambda 5007$ emission. The main difference between our 3D reconstruction and those of Vogt \& Dopita (2011) is increased spatial resolution by a factor of $\approx 2-3$ with comparable kinematic resolution, and deeper exposures sensitive to fainter emission and finer structure. Our study also leverages the emission from both lines of the [O III] doublet, allowing for more secure detections of true O-rich knots and an increased ability to more easily disentangle complex, multi-velocity component knots.

Figure 9 shows various projections of N132D, which illustrate the remnant's broken torus structure. The bulk ejecta of N132D exhibit a blue-shifted radial velocity asymmetry of -3000 to $+2300 \mathrm{~km} \mathrm{~s}^{-1}$, consistent with previous kinematic studies (e.g., Lasker 1980; Sutherland \& Dopita 1995). This velocity asymmetry of $700 \mathrm{~km} \mathrm{~s}^{-1}$ is nearly twice that determined by Vogt \& Dopita (2011), and is likely due to our more sensitive and spatially-complete survey detecting additional high-velocity knots.

\subsubsection{Best-Fitting Torus}

To quantitatively characterize the toroidal structure, we fit a torus model to the kinematic reconstruction of N132D. We fix the major radius to the best-fit semicircle radius of $1745 \mathrm{~km} \mathrm{~s}^{-1}$, as determined in Section 4.1. In order to constrain the inclination of the torus and its minor radius, we employ a Monte Carlo (MC) approach to find the minimum volume torus that contains the maximum number of main shell points, excluding emission associated with the RK. First, for a fixed minor radius, we randomly select a set of inclination angles, $\theta_{x}, \theta_{y}, \theta_{z}$, each between 0 and $360^{\circ}$ and calculate the percentage of points contained within the bestfitting torus. This process is then repeated for a large number $\left(\sim 10^{5}\right)$ of individual trials. We then choose the best-fitting torus inclination as the set of inclination angles that maximizes the percentage of points contained within the torus. Once a torus inclination is determined, we vary the value of the minor radius between 400 and $1500 \mathrm{~km} \mathrm{~s}^{-1}$ in steps of $10 \mathrm{~km} \mathrm{~s}^{-1}$ and for each value of minor radius, we again calculate the percentage of points contained within the best-fitting torus.

We define the minor radius of the torus such that it contains $75 \%$ of the main shell ejecta points by interpolating a minor radius that corresponds to $75 \%$ of enclosed points. This approach disfavors scattered material that does not strictly follow the torus distribution (highlighted in green in Figure 10). To estimate uncertainties, we repeat this calculation using an MC method, where the uncertainty of each $3 \mathrm{D}$ velocity point is modeled as a Gaussian with a width correspond to its velocity error and then for each point, a value is randomly chosen. The final value and uncertainties are reported as the median and upper and lower quartile values. We find a minor radius of $1028_{-25}^{+54} \mathrm{~km} \mathrm{~s}^{-1}$, which corresponds to a total width of $\sim 2060 \mathrm{~km} \mathrm{~s}^{-1}$, or $4.9 \mathrm{pc}$ in physical extent. With respect to a unit normal vector 

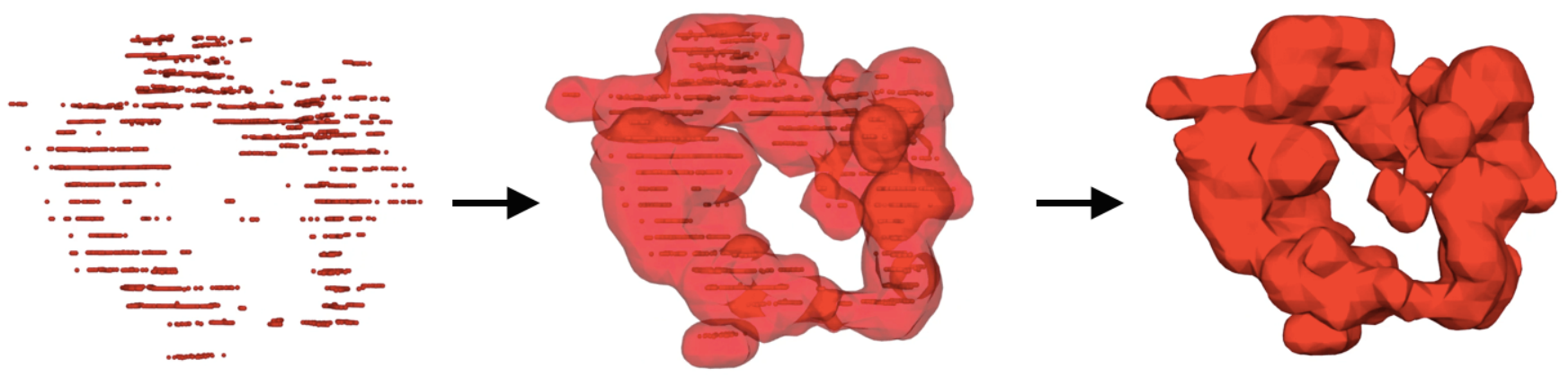

Figure 8. Surface reconstruction process for the oxygen-emitting ejecta in N132D. The three steps shown illustrate the process from initial point cloud measured spectroscopically to the surface model derived using a Marching Cubes algorithm and then retopologization with Catmull-Clark smoothing. An animation of this surface reconstruction can be found in the supporting materials of this paper.
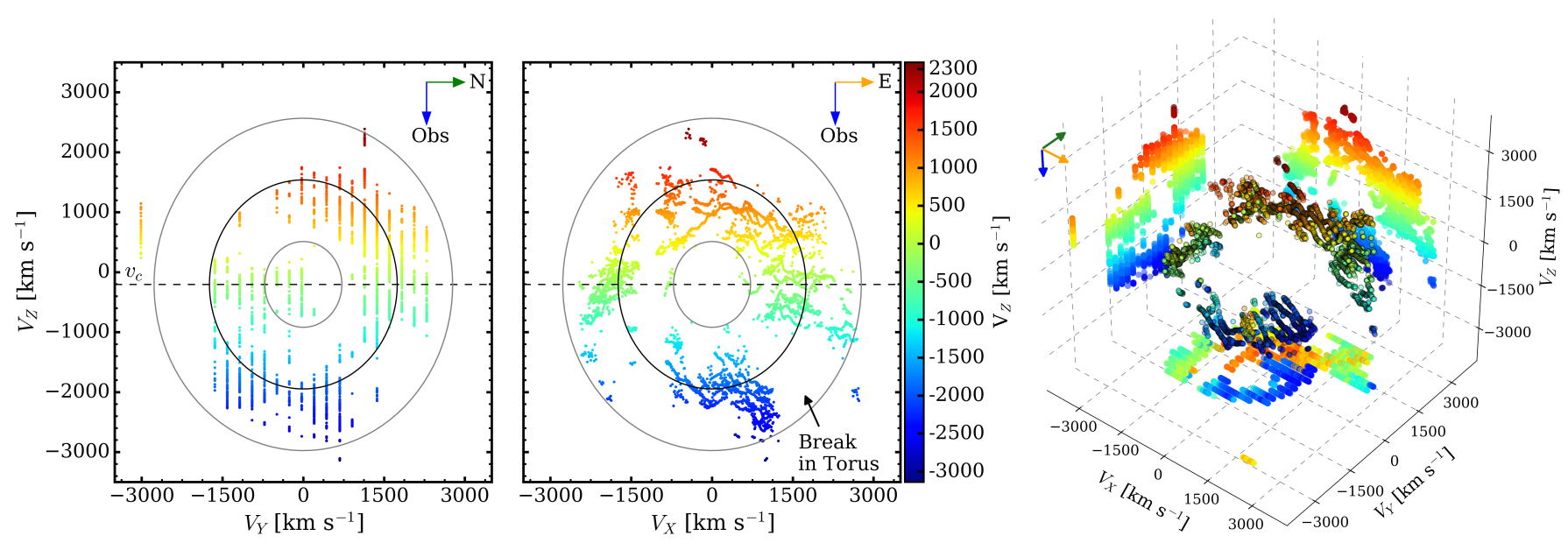

Figure 9. Projections in the $V_{Y^{-}} V_{Z}$ (left) and $V_{X}-V_{Z}$ planes (middle). The best-fit torus is overlaid with major and minor radius shown in black and gray, respectively. Three-dimensional velocity space plot with projections in all three planes (right).

in the plane of the sky oriented from the remnant to the observer, the normal vector from the torus' equatorial plane is inclined $28^{\circ} \pm 5^{\circ}$ to the line of sight. Our measurement of the inclination angle is consistent with that of Vogt \& Dopita (2011), who estimated $\sim 25^{\circ}$ via visual inspection.

There are notable deviations from a simple torus model. Perhaps most prominent is that the vertical structure of N132D varies with azimuthal angle. Specifically, the redshifted material is elevated above the midplane of the torus, while the blue-shifted side is below the mid-plane. We discuss these deviations further in Section 5.

We loaded the data into our Virtual Reality Laboratory (VR Lab; McGraw et al., in preparation) and explored the 3D morphology of N132D for evidence of ejecta substructure in the form of rings or bubbles. For instance, multi-wavelength reconstructions of Cas A have revealed large-scale coherent rings and bubbles of ejecta substructure, indicative of a bubble-like interior powered by radioactive ${ }^{56} \mathrm{Ni}$-rich ejecta (DeLaney et al. 2010; Milisavljevic \& Fesen 2013, 2015; Wongwathanarat et al. 2017). Other SNRs, including E0102 and 3C58, also exhibit ring-like structures to varying degrees (Finkelstein et al. 2006; Milisavljevic 2016; Lopez \& Fesen 2018). We were unable to conclusively identify any such substructure in N132D. However, an incomplete ring is seen toward the blue-shifted side of N132D and is composed of diffuse O-rich material that deviates from the larger torus-shaped morphology. This feature has a radius of $1000 \mathrm{~km} \mathrm{~s}^{-1}$, which is approximately $60 \%$ of the torus radius and is labeled in the lower left panel of Figure 7.

\subsubsection{Runaway Knot}

With respect to a unit normal vector from the torus' equatorial plane, the RK is inclined by approximately $82^{\circ} \pm 2^{\circ}$. The RK has a median Doppler velocity of $\sim 820 \mathrm{~km} \mathrm{~s}^{-1}$ and a high median total space velocity of 
- Main shell torus $\triangle \quad$ Diffuse, non-toroidal material $\quad$ Runaway knot
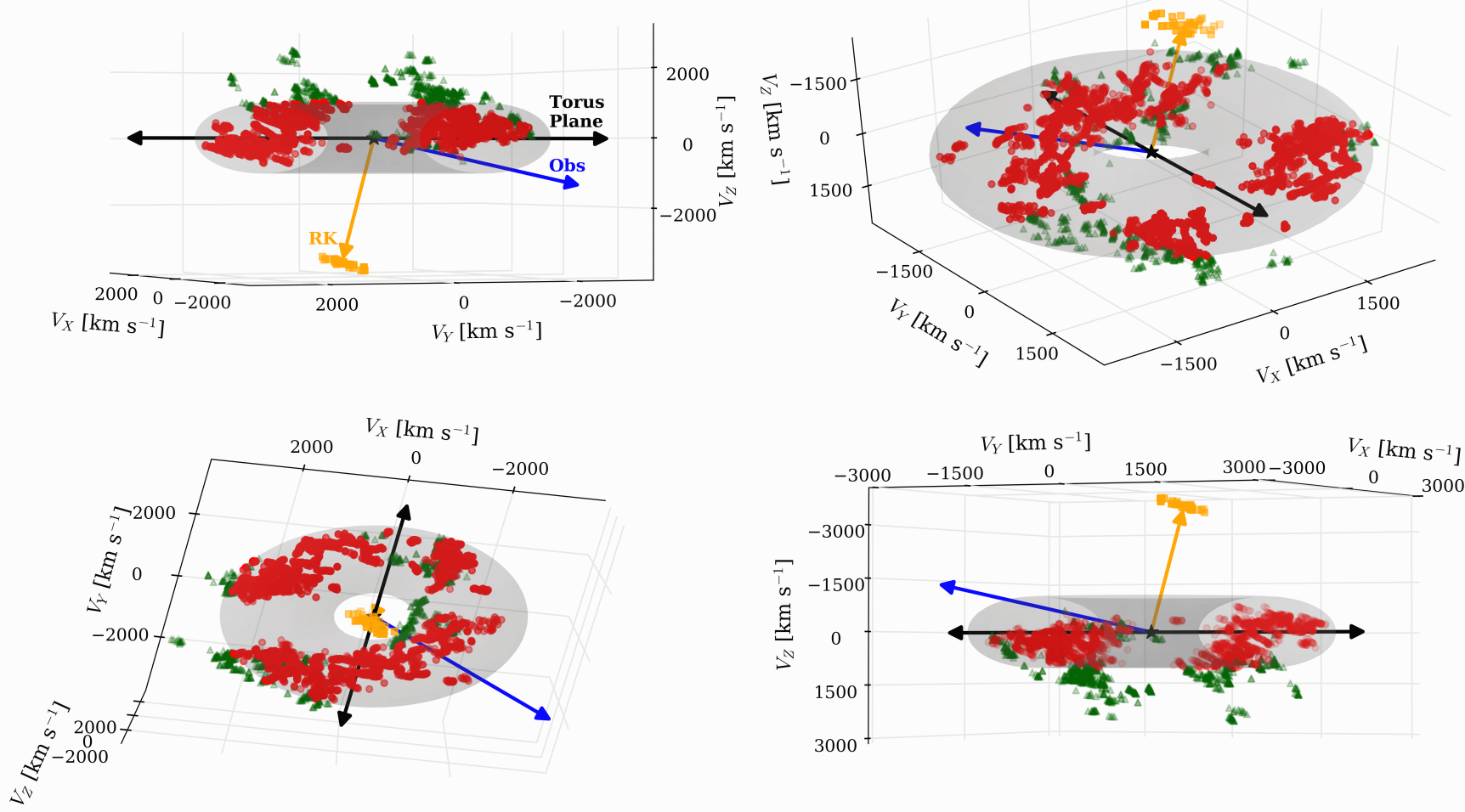

Figure 10. Various perspectives of the best-fitting torus. The top left panel shows an edge-on view, while the top right panel shows a $45^{\circ}$ view. A top-down view is shown in the bottom left and the bottom right panel is another edge-on view. Main-shell ejecta that are well-fitted by a torus are labeled in red and diffuse, scattered material that do not strictly follow a torus distribution are shown in green. The runaway knot is shown in orange.

$3650 \mathrm{~km} \mathrm{~s}^{-1}$. This is approximately twice the velocity of the bulk ejecta traveling at $1745 \mathrm{~km} \mathrm{~s}^{-1}$.

The RK is also in close proximity with a point sourcelike X-ray enhancement in the south-east of the remnant. Figure 11 shows a Chandra image in the 0.35$8.0 \mathrm{keV}$ band from $89 \mathrm{ks}$ of archival data (Borkowski et al. 2007) with our O-rich ejecta overlaid. This X-ray enhancement is located only $\sim 5^{\prime \prime}$ from the RK and is also close to a bright filament of X-ray emission. Offsets between optical and X-ray emission in SNRs are not uncommon (Patnaude \& Fesen 2014) and are associated with differences in temperature and density, as optical and X-ray emission arises from plasmas with different physical conditions. The exact relationship between the X-ray bright knot and RK (as well as the bright X-ray filament) is not clear, but our data suggest a physical association.

This X-ray enhancement is the brightest knot observed in N132D in the $0.35-8.0 \mathrm{keV}$ band, while our optical spectrum of the RK shows only weak [O III] $\lambda \lambda 4959,5007$ emission. A spectral analysis of the Chandra data indicates that this knot is enhanced in $\mathrm{Si}$ $\left(0.80_{-0.08}^{+0.14}\right)$ and slightly enhanced in $\mathrm{S}\left(0.55_{-0.14}^{+0.14}\right)$, but has a lower $\mathrm{O}$ abundance $\left(0.10_{-0.10}^{+0.33}\right)$. This contrasts with regions near the forward shock that are well-fitted with abundances of $0.4 \times$ Solar, which is typical of the ISM in the LMC. However, a definitive assessment of both the chemical abundances of this X-ray enhancement and its relationship with the RK will require X-ray data with at least an order of magnitude improvement in spectral resolution and comparable or better spatial resolution than the existing Chandra data.

\section{DISCUSSION}

Linking the kinematic and chemical characteristics of SNRs to the explosion processes associated with their parent $\mathrm{SNe}$ is rapidly growing in interest (Gabler et al. 2017; Milisavljevic \& Fesen 2017; Wongwathanarat et al. 2017; Ferrand et al. 2019). However, there are many challenges to understanding the manifold morphologies of SNRs that reflect the diversity of their progenitor star systems, prior mass loss, and environmental conditions (including, e.g., density and metallicity) that can lead to conflicting interpretations (see, e.g., Lopez et al. 2013 vs. Zhou \& Vink 2018; Broersen et al. 2014 vs. Gvaramadze et al. 2017). Typically, distributions of elements at X-ray wavelengths have been used as a classification 


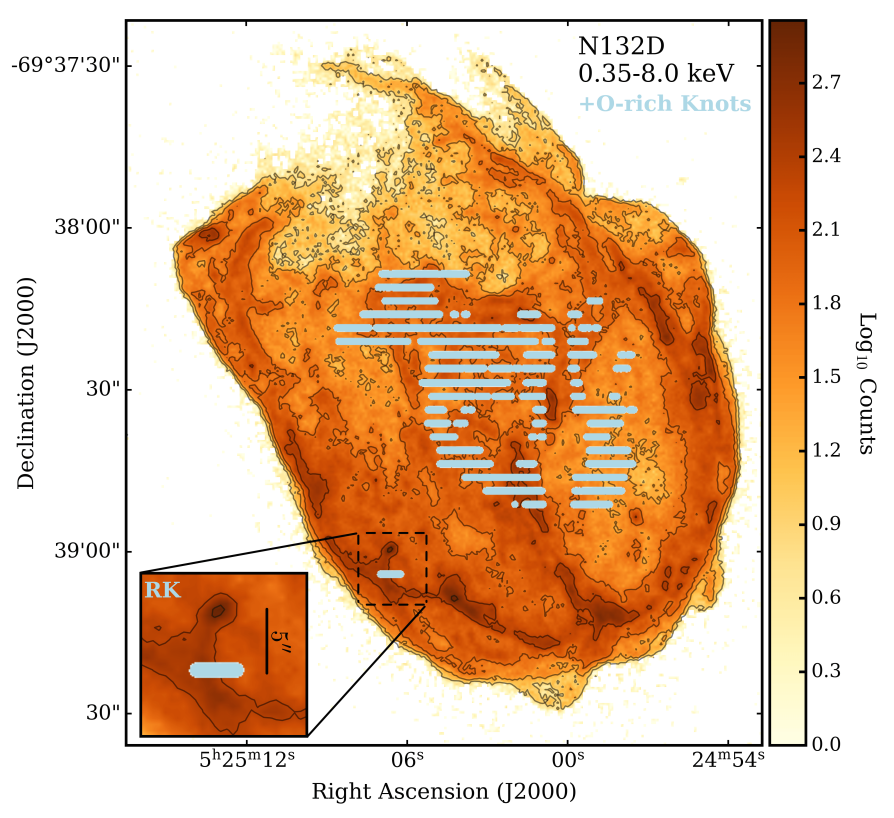

Figure 11. Chandra Advanced CCD Imaging Spectrometer S-array (ACIS-S) image of counts per pixel in N132D in the 0.35-8.0 keV band with O-rich optical ejecta overlaid. Each pixel is $0.5^{\prime \prime}$. Inset shows the $5^{\prime \prime}(\sim 1.2 \mathrm{pc})$ offset between point source-like X-ray emission and the RK.

diagnostic, and as a way to make inferences about explosion energies, nucleosynthesis yields, and density of the surrounding material (Lopez \& Fesen 2018). Recent advances of this strategy include use of Fe-K line luminosities and energy centroids (Yamaguchi et al. 2014; Patnaude et al. 2015) and mapping the distribution of ejecta with respect to remnant compact objects (Fesen et al. 2006; Katsuda et al. 2018; Holland-Ashford et al. 2019). Light echo spectroscopy has made it possible to directly connect SNRs with their parent SN classifications (Krause et al. 2008a,b; Rest et al. 2008) and to observe the explosion from multiple lines of sight (Rest et al. 2011).

Most investigations of SNRs characterize them as twodimensional projections observed on the sky. However, dense spectroscopic mapping at optical (Vogt \& Dopita 2010; Milisavljevic \& Fesen 2013), near-infrared (Kjær et al. 2010; DeLaney et al. 2010; Milisavljevic \& Fesen 2015; Larsson et al. 2016), X-ray (DeLaney et al. 2010; Grefenstette et al. 2017), and radio wavelengths (Abellán et al. 2017) has made it possible to develop $3 \mathrm{D}$ reconstructions of SN ejecta morphology. These developments have motivated substantial efforts to link explosions to their remnants with end-to-end hydrodynamic / magnetohydrodynamic models (Orlando et al.
2016, 2019). Our kinematic reconstruction of N132D contributes testable constraints for such simulations.

Many simulations have focused on explosion asymmetry introduced at the time of core-collapse by neutrinos, fluid instabilities, rotation, and magnetic fields into spherically symmetric progenitor stars and environments (Woosley \& Janka 2005; Janka 2012). However, observations and theoretical predictions have motivated additional considerations (Margutti et al. 2014; Couch \& O'Connor 2014; Nakamura et al. 2015; Summa et al. 2016; McDowell et al. 2018). It now seems likely that progenitor structure is significantly perturbed by currently poorly understood terminal phases of stellar evolution (Arnett \& Meakin 2011; Smith \& Arnett 2014), and revisions to our understanding of mass loss and observations of a high binary fraction among O-type stars (Smith 2014) suggest circumstellar environments that are not spherically symmetric (Schwarz \& Pringle 1996; Ryder et al. 2004). Below we discuss these issues in the context of the observed morphology of N132D and attempt to discern whether the large-scale structure is reflective of the original explosion kinematics and dispersal of elements, or instead has been more strongly influenced by interaction with mass loss of the progenitor star prior to core collapse.

\subsection{Geometry of O-rich Knots and Large-scale Velocity Asymmetry}

The dominant large-scale coherent structure of N132D is a distorted torus with a broken circumference. Cas A has long been known to have a general torus-like distribution of ejecta (Markert et al. 1983; DeLaney et al. 2010) with ring- and bubble-like substructure (Lawrence et al. 1995; Milisavljevic \& Fesen 2013, 2015). It is unclear whether this torus distribution is intrinsic to the explosion or is the result of interaction with an inhomogenous environment. DeLaney et al. (2010) argue that the ejecta morphology is the result of a flattened explosion where the highest velocity ejecta were expelled in a thick torus tilted not far off the plane of the sky in a number of large-scale pistons. However, high resolution mapping of Cas A at infrared wavelengths show deviations from a strict torus distribution (Isensee et al. 2010), questioning this interpretation (Milisavljevic \& Fesen 2013). Later, Milisavljevic \& Fesen (2015) mapped photoionized inner ejecta, as revealed in [S III], that were also inconsistent with a flattened explosion.

The break in the torus of N132D is not unlike discontinuities observed in other remnants. In Cas A, a conspicuous rupture in the northeastern portion of main shell ejecta located at the base of high-velocity material has persisted over decades of observations (Baade 

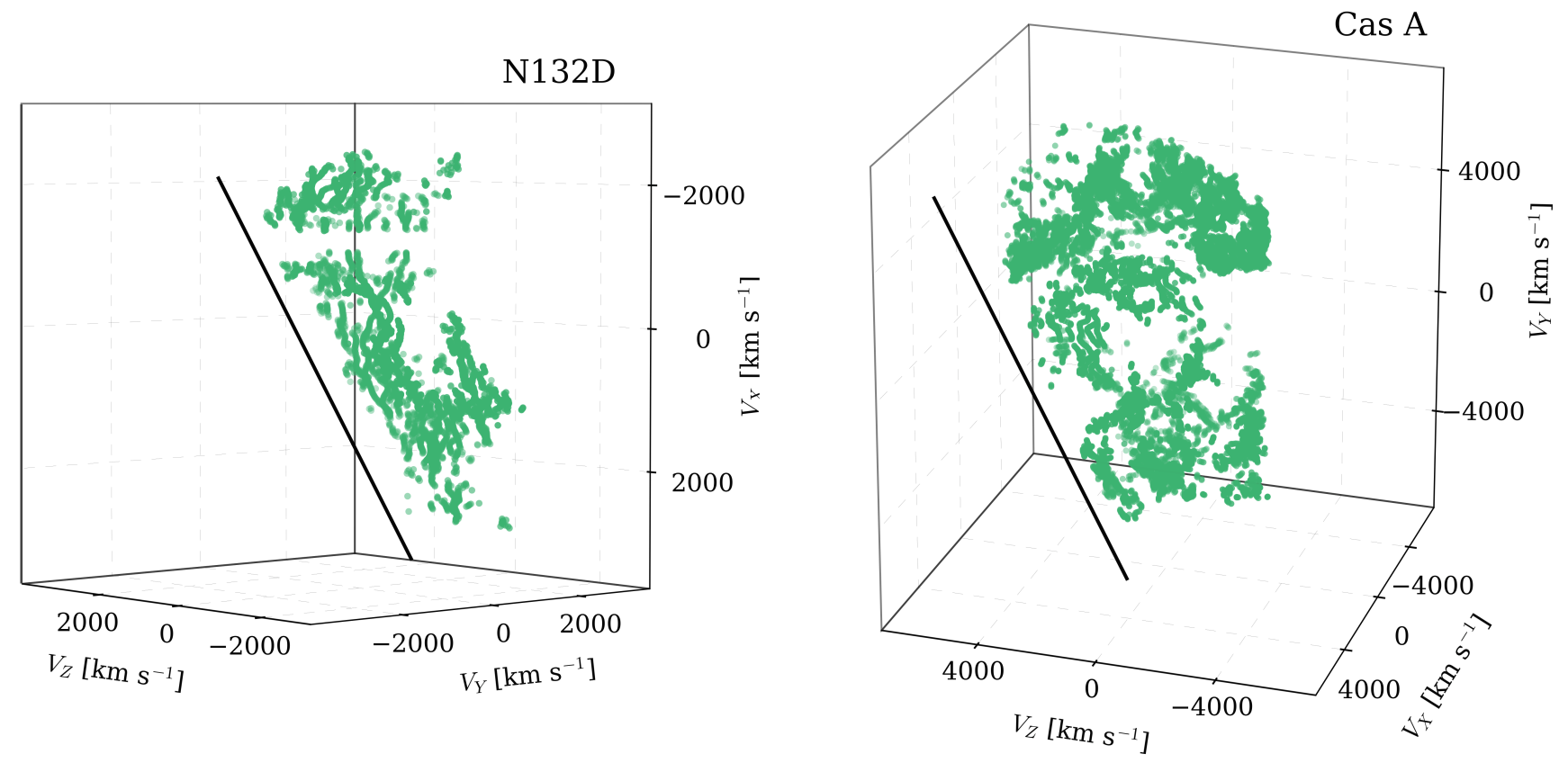

Figure 12. N132D (left) and Cas A (right) rotated at angles to illustrate their similar flat-sided torus distributions of main shell ejecta. The black lines indicate an abrupt cutoff in ejecta at a boundary defined by an angled plane. Cas A data are taken from Milisavljevic \& Fesen (2013).

\& Minkowski 1954; Patnaude \& Fesen 2014). A similar rupture-like region is observed in E0102, but is not known to be associated high-velocity ejecta (Finkelstein et al. 2006). These two remnants exhibit additional substructure in the way of rings and bubbles that N132D does not (Eriksen et al. 2001; Milisavljevic \& Fesen 2015), which may be attributable to N132D's advanced age compared to Cas A (age $\approx 340$ yr; Fesen et al. 2006) and E0102 (age $\approx 1500-2000$ yr; Finkelstein et al. 2006).

The Doppler velocity asymmetry of N132D is not uncommon in SNRs. Cas A has a -4000 to $+6000 \mathrm{~km} \mathrm{~s}^{-1}$ radial velocity asymmetry in its optical ejecta (Milisavljevic \& Fesen 2013), and E0102 has a velocity asymmetry spanning -2500 to $+3500 \mathrm{kms}^{-1}$ (Vogt \& Dopita 2010). Reed et al. (1995) argued that the velocity asymmetry in Cas A was due to an inhomogeneous environment. If the density of the surrounding medium is greater on the blueshifted near side, forward expansion is inhibited and results in an apparently redshifted COE. However, DeLaney et al. (2010) and Isensee et al. (2010) concluded that structural differences between the front and back interior surfaces could best be understood as having originated in asymmetries present in the explosion. In support of this view, the observed distribution of radioactive ${ }^{44} \mathrm{Ti}$ measured by NuSTAR, which should not be strongly influenced by the SN interaction with its environment, has a bulk line-of-sight Doppler velocity of 1100-3000 $\mathrm{km} \mathrm{s}^{-1}$ (Grefenstette et al. 2014).

An abrupt cutoff in the ejecta along a plane in the redshifted side of N132D was discovered while exploring the remnant in the VR Lab. The distribution is not unlike that observed in Cas A. In Figure 12, we show N132D and Cas $\mathrm{A}$ rotated at angles that highlight this shared morphology. Milisavljevic \& Fesen (2013) reported that the rear-facing ejecta of Cas A cutoff abruptly along an angled plane, while the front-facing ejecta do not. This sharp cutoff may be most easily understood as the result of interaction with an inhomogeneous environment. However, rotation and interaction with a binary companion can also produce non-spherical mass loss scenarios (Langer 2012).

\subsection{High-Velocity Material}

Our survey, which covers the entire $\sim 3^{\prime} \times 3^{\prime}$ field of N132D, was unable to uncover any high-velocity material in addition to the previously discovered RK. Given that (1) our survey missed $33 \%$ of the remnant (Section 2), (2) was only sensitive to [O III] $\lambda \lambda 4959,5007$ line emission, and (3) the RK is a relatively faint region of gas, it remains unclear if the RK is indeed an isolated knot or if a greater population of high-velocity material is present. The RK's close proximity to localized $\mathrm{X}$-ray emission enriched in $\mathrm{Si}$ and $\mathrm{S}$ suggests that emission lines associated with these elements may be better suited for detection. 
The RK may have a similar origin to the high-velocity ejecta in Cas A, which travel at speeds of upwards of $15,000 \mathrm{~km} \mathrm{~s}^{-1}$ (approximately three times faster than the main shell ejecta) and are enriched in S, Si, Ar, and Ca emission (Fesen \& Gunderson 1996). Although their broadness and kinetic energy argue against the Cas A SN being a jet-induced explosion (Laming et al. 2006; Fesen \& Milisavljevic 2016), the jets are kinematically and chemically distinct from the rest of the remnant (Fesen 2001; Hwang et al. 2004). This may reflect an origin in a jet-like mechanism that accelerated interior material from a Si-, S-, Ar-, and Ca-rich region near the progenitor's core up through the mantle and $\mathrm{H}-$, He-, $\mathrm{N}$-, and O-rich outer layers with velocities that greatly exceeded that of the rapidly expanding photosphere.

The RK is also not unlike ejecta clumps or "bullets" located in the northeastern edge of the Vela remnant, which exhibit a high Si abundance, in contrast to the other observed ejecta fragments that show enhanced $\mathrm{O}$, Ne, and Mg abundances (Aschenbach et al. 1995; Katsuda \& Tsunemi 2006). Recently, another ejecta fragment located opposite to shrapnel A with respect to the center of the shell was found in the southwestern boundary of the remnant, strongly suggesting a Si-rich jet-counterjet structure (García et al. 2017).

Our findings are possibly relevant to the continuum of explosion energies, extending from broad-lined Type Ic SNe associated with gamma-ray bursts to more ordinary Type Ib/c SNe, that appears to exist (Mazzali et al. 2008; Margutti et al. 2014; Milisavljevic et al. 2015; Sobacchi et al. 2017; Barnes et al. 2018) and that may be associated with energy input from compact objects formed during core-collapse. The connection may also extend to superluminous SNe (Milisavljevic et al. 2013; Mazzali et al. 2014; Metzger et al. 2015; Nicholl et al. 2016; Milisavljevic et al. 2018). The implication is that a wide variety of jet activity may potentially be occurring at energies that participate in but do not necessarily drive explosions (Fesen \& Milisavljevic 2016; Ertl et al. 2019). At extragalactic distances, explosions with weak or choked jets that lack sizeable relativistic ejecta are indistinguishable from events without jets (Lazzati et al. 2012). Only through resolved inspection of nearby supernova remnants can the presence of these structures be identified. Origins in the diversity of jet activity may be related to the type of compact object formed (neutron star vs. magnetar vs. black hole) and whether the central engine activity becomes choked before the jet is able to pierce through the stellar envelope.

\section{CONCLUSIONS}

We have presented a 3D kinematic reconstruction of optical emission from oxygen-rich ejecta in SNR N132D based on radial velocity measurements extracted from long-slit spectra. Based on this high spatial and kinematic resolution data set, we conclude the following:

1. We confirm the findings of previous kinematic studies of N132D that show the bulk of the remnant's optically bright oxygen-rich ejecta are arranged in a torus-like geometry. We find that this torus-shaped material is tilted by $28^{\circ}$ with respect to the plane of the sky, has a radius of $4.4 \mathrm{pc}$ ( $\left.D_{\mathrm{LMC}} / 50 \mathrm{kpc}\right)$, exhibits a blue-shifted radial velocity asymmetry of -3000 to $+2300 \mathrm{~km} \mathrm{~s}^{-1}$, and has a conspicuous break in its circumference.

2. Assuming homologous expansion from the geometric center of O-rich filaments, the average expansion velocity of $1745 \mathrm{~km} \mathrm{~s}^{-1}$ translates to an age since explosion of $2450 \pm 195 \mathrm{yr}$, which is consistent with the kinematic age determined from the lower spatial resolution study by Vogt \& Dopita (2011).

3. A faint, spatially-separated "runaway knot" with total space velocity of $3650 \mathrm{~km} \mathrm{~s}^{-1}$ is nearly perpendicular to the plane of N132D's main torus and coincident with localized X-ray emission that is enhanced in Si relative to the LMC and N132D's main ejecta. The kinematic and chemical signatures suggest that the RK may have had its origin deep within the progenitor star.

4. N132D has notable kinematic and chemical similarities with Cas A. Both have a general torus morphology with a rupture, a sharp cutoff at a boundary defined by an angled plane, and highvelocity Si-enriched knots.

Our results underscore the need for SN-to-SNR simulations that take into consideration evolutionary phases and the associated mass loss that massive stars experience approaching core collapse. Our analyses strongly suggest that environment is an important contribution to the observed morphology. Further work comparing and contrasting morphology and chemical abundances of additional SNRs will provide valuable constraints for simulations attempting to model and correctly interpret the electromagnetic+gravitational wave+neutrino signals of SNe.

The authors thank the anonymous referee for valuable comments that improved both the content and presentation of this work. This work has benefited from the helpful comments of Frédéric Vogt and Robert Fesen. This 
paper includes data gathered with the 6.5 meter Magellan Telescopes located at Las Campanas Observatory, Chile. STSDAS and PyRAF are products of the Space Telescope Science Institute, which is operated by AURA for NASA. This publication makes use of data products from the Two Micron All Sky Survey, which is a joint project of the University of Massachusetts and the Infrared Processing and Analysis Center/California Institute of Technology, funded by the National Aeronautics and Space Administration and the National Science Foundation. This work is also based on observations made with the NASA/ESA Hubble Space Telescope, obtained from the Data Archive at the Space Telescope Science Institute, which is operated by the Association of Universities for Research in Astronomy, Inc., under NASA contract NAS 5-26555. These observations are associated with program 12001 . The work also incorporates data obtained from the Chandra Data Archive and observations made by the Chandra X-ray Observatory. C.J.L. acknowledges funding from the National Science Foundation Graduate Research Fellowship under Grant DGE1745303.

Facilities: Magellan:Baade(IMACS+GISMO), CXO

Software: DS9 (Joye \& Mandel 2003), IRAF/PyRAF (Tody 1986), L.A.Cosmic (van Dokkum 2001), Matplotlib (Hunter 2007), NumPy (van der Walt et al. 2011)

\section{REFERENCES}

Abellán, F. J., Indebetouw, R., Marcaide, J. M., et al.

2017, ApJL, 842, L24

Ackermann, M., Albert, A., Atwood, W. B., et al. 2016, A\&A, 586, A71

Arnett, W. D., \& Meakin, C. 2011, ApJ, 733, 78

Aschenbach, B., Egger, R., \& Trümper, J. 1995, Nature, 373,587

Baade, W., \& Minkowski, R. 1954, ApJ, 119, 206

Bamba, A., Ohira, Y., Yamazaki, R., et al. 2018, ApJ, 854, 71

Banas, K. R., Hughes, J. P., Bronfman, L., \& Nyman, L.-A. 1997, ApJ, 480, 607

Barnes, J., Duffell, P. C., Liu, Y., et al. 2018, ApJ, 860, 38

Beasley, M., Morse, J. A., Green, J. C., \& Cosmic Origins Spectrograph Science. 2004, in American Astronomical Society Meeting Abstracts, Vol. 205, American

Astronomical Society Meeting Abstracts, 101.04

Blair, W. P., Morse, J. A., Raymond, J. C., et al. 2000, ApJ, 537, 667

Borkowski, K. J., Hendrick, S. P., \& Reynolds, S. P. 2007, ApJL, 671, L45

Broersen, S., Chiotellis, A., Vink, J., \& Bamba, A. 2014, MNRAS, 441, 3040

Burrows, A., Radice, D., \& Vartanyan, D. 2019, MNRAS, 485, 3153

Catmull, E., \& Clark, J. 1998 (New York, NY, USA: ACM), 183-188. http://doi.acm.org/10.1145/280811.280992

Chen, Y., Zhang, F., Williams, R. M., \& Wang, Q. D. 2003, ApJ, 595, 227

Chevalier, R. A., \& Kirshner, R. P. 1978, ApJ, 219, 931

Clark, D. H., Tuohy, I. R., Dopita, M. A., et al. 1982, ApJ, 255,440

Couch, S. M., \& O'Connor, E. P. 2014, ApJ, 785, 123

Danziger, I. J., \& Dennefeld, M. 1976, ApJ, 207, 394
DeLaney, T., Rudnick, L., Stage, M. D., et al. 2010, ApJ, 725,2038

Dopita, M. A., Tuohy, I. R., \& Mathewson, D. S. 1981, ApJL, 248, L105

Dopita, M. A., Vogt, F. P. A., Sutherland, R. S., et al. 2018, ApJS, 237, 10

Dressler, A., Bigelow, B., Hare, T., et al. 2011, PASP, 123, 288

Eriksen, K. A., Morse, J. A., Kirshner, R. P., \& Winkler, P. F. 2001, in American Institute of Physics Conference Series, Vol. 565, Young Supernova Remnants, ed. S. S. Holt \& U. Hwang, 193-196

Ertl, T., Woosley, S. E., Sukhbold, T., \& Janka, H. T. 2019, arXiv e-prints, arXiv:1910.01641

Favata, F., Vink, J., Parmar, A. N., Kaastra, J. S., \& Mineo, T. 1997, A\&A, 324, L45

Ferrand, G., Warren, D. C., Ono, M., et al. 2019, ApJ, 877, 136

Fesen, R. A. 2001, ApJS, 133, 161

Fesen, R. A., \& Gunderson, K. S. 1996, ApJ, 470, 967

Fesen, R. A., \& Milisavljevic, D. 2016, ApJ, 818, 17

Fesen, R. A., Hammell, M. C., Morse, J., et al. 2006, The Astrophysical Journal, 645, 283.

http://stacks.iop.org/0004-637X/645/i=1/a=283

Finkelstein, S. L., Morse, J. A., Green, J. C., et al. 2006, ApJ, 641, 919

France, K., Beasley, M., Keeney, B. A., et al. 2009, ApJL, 707, L27

Gabler, M., Janka, H.-T., \& Wongwathanarat, A. 2017, in IAU Symposium, Vol. 331, Supernova 1987A:30 years later - Cosmic Rays and Nuclei from Supernovae and their Aftermaths, ed. A. Marcowith, M. Renaud, G. Dubner, A. Ray, \& A. Bykov, 141-147 
Gal-Yam, A. 2017, Observational and Physical Classification of Supernovae, 195

García, F., Suárez, A. E., Miceli, M., et al. 2017, A\&A, 604, L5

Goss, W. M., Shaver, P. A., Zealey, W. J., Murdin, P., \& Clark, D. H. 1979, MNRAS, 188, 357

Grefenstette, B. W., Harrison, F. A., Boggs, S. E., et al. 2014, Nature, 506, 339

Grefenstette, B. W., Fryer, C. L., Harrison, F. A., et al. 2017, ApJ, 834, 19

Gvaramadze, V. V., Langer, N., Fossati, L., et al. 2017, Nature Astronomy, 1, 0116

Hendrick, S. P., Reynolds, S. P., \& Borkowski, K. J. 2005, ApJL, 622, L117

H.E.S.S. Collaboration, Abramowski, A., Aharonian, F., et al. 2015, Science, 347, 406

Holland-Ashford, T., Lopez, L. A., \& Auchettl, K. 2019, arXiv e-prints, arXiv:1904.06357

Hunter, J. D. 2007, Computing in Science and Engineering, 9,90

Hwang, U., Hughes, J. P., Canizares, C. R., \& Markert, T. H. 1993, ApJ, 414, 219

Hwang, U., Laming, J. M., Badenes, C., et al. 2004, ApJL, 615, L117

Isensee, K., Rudnick, L., DeLaney, T., et al. 2010, ApJ, 725,2059

Janka, H.-T. 2012, Annual Review of Nuclear and Particle Science, 62, 407

Janka, H.-T., Melson, T., \& Summa, A. 2016, Annual Review of Nuclear and Particle Science, 66, 341

Joye, W. A., \& Mandel, E. 2003, Astronomical Society of the Pacific Conference Series, Vol. 295, New Features of SAOImage DS9, ed. H. E. Payne, R. I. Jedrzejewski, \& R. N. Hook, 489

Katsuda, S., \& Tsunemi, H. 2006, ApJ, 642, 917

Katsuda, S., Morii, M., Janka, H.-T., et al. 2018, ApJ, 856, 18

Kirshner, R. P., \& Chevalier, R. A. 1977, ApJ, 218, 142

Kjær, K., Leibundgut, B., Fransson, C., Jerkstrand , A., \& Spyromilio, J. 2010, A\&A, 517, A51

Krause, O., Birkmann, S. M., Usuda, T., et al. 2008a, Science, 320, 1195

Krause, O., Tanaka, M., Usuda, T., et al. 2008b, Nature, 456,617

Kuroda, T., Kotake, K., Hayama, K., \& Takiwaki, T. 2017, ApJ, 851, 62

Laming, J. M., Hwang, U., Radics, B., Lekli, G., \& Takács, E. 2006, ApJ, 644, 260

Langer, N. 2012, ARA\&A, 50, 107
Larsson, J., Fransson, C., Spyromilio, J., et al. 2016, The Astrophysical Journal, 833, 147.

http://stacks.iop.org/0004-637X/833/i=2/a=147

Lasker, B. M. 1978, ApJ, 223, 109

-. 1980, ApJ, 237, 765

Lawrence, S. S., MacAlpine, G. M., Uomoto, A., et al. 1995, AJ, 109, 2635

Lazendic, J. S., \& Slane, P. O. 2006, ApJ, 647, 350

Lazzati, D., Morsony, B. J., Blackwell, C. H., \& Begelman, M. C. 2012, ApJ, 750, 68

Lopez, L. A., \& Fesen, R. A. 2018, SSRv, 214, 44

Lopez, L. A., Ramirez-Ruiz, E., Castro, D., \& Pearson, S. 2013, ApJ, 764, 50

Lorensen, W. E., \& Cline, H. E. 1987, SIGGRAPH Comput. Graph., 21, 163.

http://doi.acm.org/10.1145/37402.37422

Margutti, R., Milisavljevic, D., Soderberg, A. M., et al. 2014, ApJ, 780, 21

Markert, T. H., Canizares, C. R., Clark, G. W., \& Winkler, P. F. 1983, ApJ, 268, 134

Mathewson, D. S., Dopita, M. A., Tuohy, I. R., \& Ford, V. L. 1980, ApJL, 242, L73

Mazzali, P. A., McFadyen, A. I., Woosley, S. E., Pian, E., \& Tanaka, M. 2014, MNRAS, 443, 67

Mazzali, P. A., Valenti, S., Della Valle, M., et al. 2008, Science, 321, 1185

McDowell, A. T., Duffell, P. C., \& Kasen, D. 2018, ApJ, 856,29

Metzger, B. D., Margalit, B., Kasen, D., \& Quataert, E. 2015, MNRAS, 454, 3311

Milisavljevic, D. 2016, IAU Focus Meeting, 29B, 225

Milisavljevic, D., \& Fesen, R. A. 2013, ApJ, 772, 134

—. 2015, Science, 347, 526

- 2017, The Supernova - Supernova Remnant Connection, 2211

Milisavljevic, D., Fesen, R. A., Gerardy, C. L., Kirshner, R. P., \& Challis, P. 2010, ApJ, 709, 1343

Milisavljevic, D., Patnaude, D. J., Chevalier, R. A., et al. 2018, ApJL, 864, L36

Milisavljevic, D., Soderberg, A. M., Margutti, R., et al. 2013, ApJL, 770, L38

Milisavljevic, D., Margutti, R., Parrent, J. T., et al. 2015, ApJ, 799, 51

Morse, J. A., Winkler, P. F., \& Kirshner, R. P. 1995, AJ, 109,2104

Morse, J. A., Blair, W. P., Dopita, M. A., et al. 1996, AJ, 112,509

Müller, B., \& Janka, H. T. 2015, MNRAS, 448, 2141

Murdin, P., \& Clark, D. H. 1979, MNRAS, 189, 501 
Nakamura, K., Takiwaki, T., Kuroda, T., \& Kotake, K. 2015, PASJ, 67, 107

Nicholl, M., Berger, E., Margutti, R., et al. 2016, ApJL, 828, L18

O'Connor, E. P., \& Couch, S. M. 2018, ApJ, 865, 81

Orlando, S., Miceli, M., Pumo, M. L., \& Bocchino, F. 2015, ApJ, 810, 168

—. 2016, ApJ, 822, 22

Orlando, S., Miceli, M., Petruk, O., et al. 2019, A\&A, 622, A73

Panagia, N., Gilmozzi, R., Macchetto, F., Adorf, H.-M., \& Kirshner, R. P. 1991, ApJL, 380, L23

Pannuti, T. G., Rho, J., Borkowski, K. J., \& Cameron, P. B. 2010, AJ, 140, 1787

Pannuti, T. G., Rho, J., Kargaltsev, O., et al. 2017, ApJ, 839, 59

Park, S., Hughes, J. P., Burrows, D. N., et al. 2003, ApJL, 598, L95

Patnaude, D. J., \& Fesen, R. A. 2014, ApJ, 789, 138

Patnaude, D. J., Lee, S.-H., Slane, P. O., et al. 2015, ApJ, 803, 101

Reed, J. E., Hester, J. J., Fabian, A. C., \& Winkler, P. F. 1995, ApJ, 440, 706

Rest, A., Matheson, T., Blondin, S., et al. 2008, ApJ, 680, 1137

Rest, A., Foley, R. J., Sinnott, B., et al. 2011, ApJ, 732, 3

Ryder, S. D., Sadler, E. M., Subrahmanyan, R., et al. 2004, MNRAS, 349, 1093

Sano, H. in prep.

Sano, H., Fujii, K., Yamane, Y., et al. 2017, in American Institute of Physics Conference Series, Vol. 1792, 6th International Symposium on High Energy Gamma-Ray Astronomy, 040038

Schwarz, D. H., \& Pringle, J. E. 1996, MNRAS, 282, 1018
Sharda, P., Gaetz, T. J., Kashyap, V. L., \& Plucinsky, P. P. 2020, submitted, ApJ

Skrutskie, M. F., Cutri, R. M., Stiening, R., et al. 2006, AJ, 131,1163

Smith, N. 2014, Annual Review of Astronomy and Astrophysics, 52, 487. https:

//doi.org/10.1146/annurev-astro-081913-040025

Smith, N., \& Arnett, W. D. 2014, ApJ, 785, 82

Sobacchi, E., Granot, J., Bromberg, O., \& Sormani, M. C. 2017, MNRAS, 472, 616

Storey, P. J., \& Zeippen, C. J. 2000, MNRAS, 312, 813

Summa, A., Hanke, F., Janka, H.-T., et al. 2016, ApJ, 825, 6

Sutherland, R. S., \& Dopita, M. A. 1995, ApJ, 439, 365

Tappe, A., Rho, J., \& Reach, W. T. 2006, ApJ, 653, 267

Tody, D. 1986, in Society of Photo-Optical Instrumentation Engineers (SPIE) Conference Series, Vol. 627,

Proc. SPIE, ed. D. L. Crawford, 733

van der Marel, R. P., Alves, D. R., Hardy, E., \& Suntzeff, N. B. 2002, AJ, 124, 2639

van der Walt, S., Colbert, S. C., \& Varoquaux, G. 2011,

Computing in Science and Engineering, 13, 22

van Dokkum, P. G. 2001, PASP, 113, 1420

Vogt, F., \& Dopita, M. A. 2010, ApJ, 721, 597

—. 2011, Ap\&SS, 331, 521

Westerlund, B. E., \& Mathewson, D. S. 1966, MNRAS, 131,371

Winkler, P. F., \& Kirshner, R. P. 1985, ApJ, 299, 981

Wongwathanarat, A., Janka, H.-T., Müller, E., Pllumbi, E., \& Wanajo, S. 2017, ApJ, 842, 13

Woosley, S., \& Janka, T. 2005, Nature Physics, 1, 147

Xiao, X., \& Chen, Y. 2008, Advances in Space Research, 41,416

Yamaguchi, H., Badenes, C., Petre, R., et al. 2014, ApJL, 785, L27

Zhou, P., \& Vink, J. 2018, A\&A, 615, A150 